九州大学学術情報リポジトリ

Kyushu University Institutional Repository

\title{
A Statistical Analysis of Sputtering Parameters on Superconducting Properties of Niobium Thin Fi Im
}

Gaurav Gupta

Department of Mechanical Engineering, Amity University U. P.

R. K. Tyagi

Department of Mechanical Engineering, Amity University U. P.

S. K. Rajput

Mechanical Engineering Department, Bundelkhand Institute of Engineering and Technology

https://doi.org/10.5109/4372259

出版情報: Evergreen. 8 (1)，pp.44-50，2021-03. Transdisciplinary Research and Education Center for Green Technologies, Kyushu University バージョン:

権利関係 : 


\title{
A Statistical Analysis of Sputtering Parameters on Superconducting Properties of Niobium Thin Film
}

\author{
Gaurav Gupta $^{1 *}$, R. K. Tyagi ${ }^{1}$, S. K. Rajput ${ }^{2}$ \\ ${ }^{1}$ Department of Mechanical Engineering, Amity University U. P., Noida-201303 \\ ${ }^{2}$ Mechanical Engineering Department, Bundelkhand Institute of Engineering and Technology, Jhansi 284128, \\ India
}

*Author to whom correspondence should be addressed:

E-mail: 15.gaurav@gmail.com

(Received October 24, 2020; Revised December 30, 2020; accepted January 29, 2021).

\begin{abstract}
With everchanging technology and constantly increasing demand of energy, superconducting materials can play a crucial role as by offering lowest possible electrical resistance. In this quest, Niobium $(\mathrm{Nb})$ thin films synthesized by magnetron sputtering has been analyzed at diverse sputtering constraints. Different parameters as Nitrogen $\left(\mathrm{N}_{2}\right)$ partial pressure, power densities, magnetization are explored by Usadel equation. Usadel equation integrating BardeenCooper-Schrieffer theory of superconductivity, analyzed dependence of superconducting transition temperature on varied sputtering parameters by computational technique. It has been found that Nioubium Nitride $(\mathrm{NbN})$ operating at elevated temperature exhibits superconductive properties. Optimal growth is found at $\mathrm{N}_{2}$ partial pressure $\sim 15$ Standard Cubic Centimeters per Minute (SCCM) where power densities ranges from 200 to $500 \mathrm{~W}$ at a critical temperature of $14.5 \mathrm{~K}$.
\end{abstract}

Keywords: Sputtering; Superconducting; Thin film; Growth

\section{Introduction}

For state of art scientific research, potential of new superconducting materials are explored which can operate at elevated temperatures and presents new industrial applications where Niobium is found extremely suitable ${ }^{1-}$ 3). Thin films grown of superconductor materials on suitable substrate presents possibility of enormous range of devices exhibiting superconducting properties at varied operational ranges viz. temperature, magnetic field, voltage etc. ${ }^{4-6)}$. In today's world of energy deprived world, it is of great interest to identify or develop engineered materials capable of high performance applications at reduced energy consumption ${ }^{7}$. Thin film growth, often multilayer ${ }^{4)}$, of superconducting material presents a promising technique of obtaining high performance superconducting cavities. Such coated superconductors in the form of superconducting nanowire single photon detectors (SNSPDs), superconducting radiofrequency (SRF) cavities, Superconductor-Insulator-Superconductor (SIS) tunnel junctions ${ }^{8-12)}$ presents a huge potential for diverse applications ranging from electronic industry to aerospace industry and industrial applications. As reduced power consumption like in power cables, magnets, motors, quantum information, quantum optics, free space laser communication, quantum electronics, particle accelerators, etc. ${ }^{3,5,13)}$.

For the above varied applications Transition Metal
Nitrides (TMN) has been widely accepted. Many studies were focused on Niobium, Titanium, Lead, Bismuth \& Tin as superconductive coating 9,14-17). For depositing thin films various techniques of sputtering are employed such as pulsed DC, pulsed laser deposition, RF and DC sputtering ${ }^{18-20)}$ chemical vapor deposition (CVD) ${ }^{11)}$ Ion Beam Sputtering ${ }^{21)}$ are used to obtain stable thin film of superconductors, also these techniques are deployed to fabricate Spintronic devices. The spintronic devices has superior performance wherein same semiconductor charge, spin of moving electron is manipulated.

Deposited superconductors on a substrate involves following steps (a) substrate preparation- involving superfinishing operations, (b) buffer / isolating layer, (c) superconducting layer (sputtering technique), (d) protective layer. ${ }^{22-24)}$. Stability of such films poses great challenge alongwith performance specially under the effect of temperatures, magnetic field, electrical resistivity etc. Researchers have been studying characteristics, homogeneity etc. of film deposited on various substrates ( $\mathrm{MgO}$, Silicon, sapphire) which shows a great dependence on technology of deposition and parameters chosen for deposition ${ }^{25,26)}$.

Matsumoto et al., ${ }^{5)}$ fabricated thin film Bi-Sr-Ca-Cu-O (BSCCO) and successfully doped $\mathrm{Bi}, \mathrm{Pb}-2223$ in stable phase using RF sputtering to investigate superconducting properties. Where an significant 
improvement of around $10.8 \mathrm{~K}$ in transition temperature $\left(\mathrm{T}^{\prime} \mathrm{c}\right)$ were observed. Xiaoyan et al. ${ }^{15)}$ obtained thin film of Niobium Titanium Nitride ( $\mathrm{NbTiN}$ ) and $\mathrm{NbN}$ on $\mathrm{Si}$ wafers and concluded that NbTiN stands out in performance (low resistivity at elevated temp., low kinetic inductance, reduced recovery time of photon response, higher switching currents $\left(\mathrm{I}_{\mathrm{sw}}\right)$, low timing jitter (TJ), kinetic inductance ) and uniformity as compared to $\mathrm{NbN}$. Tomas polakovic et al, ${ }^{8)}$ shows : Growth of film depends on concentration of gases and sputtering powers which in turn needs precise control. In DC magnetron sputtering employing $\mathrm{Nb}$ target in presence of neutralized nitrogen is capable of producing $\mathrm{NbN}$ thin films with higher $\mathrm{T}_{\mathrm{c}}$. (superconducting transition temperatures) $14.5 \mathrm{~K}$ and resistivity of $110.62 \mu \Omega . \mathrm{cm}$.

A. Shoji et al ${ }^{27)}$ finds that Reactive sputtering is capable of producing desired stable phases of $\mathrm{NbN}$ at temperatures more than $773 \mathrm{~K}$ whereas higher $\mathrm{T}^{\prime}{ }_{\mathrm{c}}$ of $\mathrm{NbN}$ film can be obtained by using methane gas in RF diode sputtering at $300 \mathrm{~K}^{28)}$ and gives high resistivity above $10^{4} \mu \Omega . \mathrm{cm}$ due to presence of columnar or granular structure ${ }^{29)}$. Also Bombarding ion at energies above $300 \mathrm{eV}$ can cause damage to structure of thin film as claimed by W. Kern et al. ${ }^{30)}$ also they showed that their exits superconducting T' at $12.5 \mathrm{~K}$ or $>12 \mathrm{~K}$ for concentration of nitrogen ranging from $7.5 \%$ to $13 \%$.

The superconducting state of $\mathrm{NbN}$ suppresses as the deposited film thickness reaches to thin film critical thickness, for superconducting thin films critical thickness is $2.7 \mathrm{~nm}$ below which $\mathrm{NbN}$ thin film are not superconducting ${ }^{31,32)}$. However, influence of sputtering parameters, primarily on thickness and other properties is still an open question and researchers are worldwide experimenting towards it. As cited above Deposition parameters and growth method has significant effect on structural properties.

Niobium Nitride presents a promising element acting as thin layer of superconducting element. Which is comparatively easy to fabricate along with large superconducting energy gap, relatively high superconducting $\left(\mathrm{T}_{\mathrm{c}}^{\prime}\right)$ temperature thus showing superconducting properties at elevated temperatures.

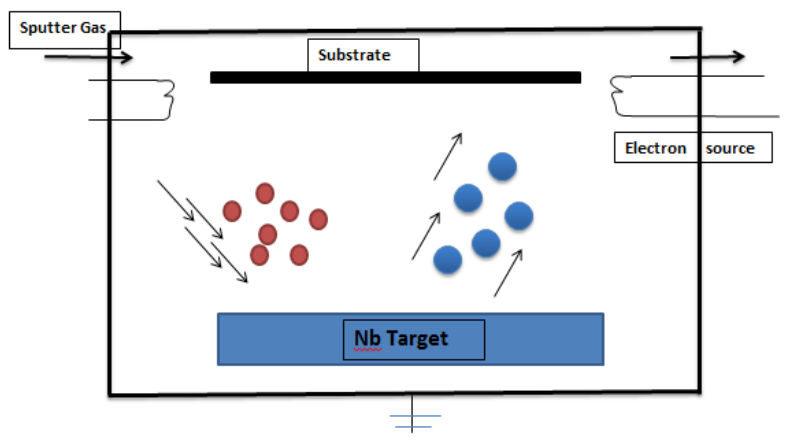

Fig. 1: Schematic picture of sputtering chamber

A magnetron sputtering device as shown in Fig. 1 is considered wherein Nitrogen plasma parameters on relative thickness of film over a substrate surface is computed.

Here in this study superconductive nature of Niobium thin film and their synthesis by Magnetron sputter deposition upon substrates at different parameters as Nitrogen $\left(\mathrm{N}_{2}\right)$ partial pressure, power densities, magnetization are investigated by computational technique using Usadel equation for a superconductive state.

\section{Materials and methods}

Magnetron sputtering method employs bombardment of target element by ions at high energy and velocity under the influence of electric and magnetic field in the presence of nitrogen plasma. Figure 1 shows device for sputtering which is considered for deposition of $\mathrm{Nb}$ as thin film with use of Nitrogen gas as plasma, ions extracted from plasma bombards target surface, as positioned very near to plasma, this bombardment results in dislodging of target atoms and is directed towards substrate and makes a thin layer over bulk material. Plasma ions spot size 2-6mm and diameter of column is taken as $4 \mathrm{~cm}$ with ion velocity derived from real frequency of waves ${ }^{33,34)}$ which further depends on electromagnetic lenses focusing. Sputtering chamber pressure is considered $2 \times 10^{-5} \mathrm{~Pa}^{8,15)}$, flow of gas 10-20 Standard Cubic Centimeters per Minute (SCCM), energies above $100 \mathrm{ev}$. Ratio of threshold energy by ions energy are adopted from Grais et. al ${ }^{35)}$. Relative thickness of film is calculated by following relation ${ }^{36}$ )

$T_{p}=h^{2}\left(T_{p 1}-\alpha h^{2} T_{p 2}\right)$

$T_{c}=T_{c 1}-\alpha T_{c 2}$

Where $\mathrm{T}_{\mathrm{p} 1}, \mathrm{~T}_{\mathrm{p} 2}, \mathrm{~T}_{\mathrm{c} 1}, \mathrm{~T}_{\mathrm{c} 2}$ is as follows: -

$$
\begin{aligned}
& T_{p 1}=2 \int_{R_{1}}^{R_{2}} R d R \int_{0}^{\pi} \frac{d \phi}{L_{0}^{5}(1-K \cos \phi)^{5 / 2}} \\
& T_{p 2}=2 \int_{R_{1}}^{R_{2}} R d R \int_{0}^{\pi} \frac{d \phi}{L_{0}{ }^{7}(1-K \cos \phi)^{7 / 2}} \\
& T_{c 1}=\int_{h}^{h+1} h d h \int_{0}^{2 \pi} R_{2} d \phi \frac{\left(R_{2}-r \cos \phi\right)}{L_{0}^{5}(1-K \cos \phi)^{5 / 2}} \\
& T_{c 2}=\int_{h}^{h+1} h d h \int_{0}^{2 \pi} R_{2} d \phi \frac{\left(R_{2}-r \cos \phi\right)^{3}}{L_{0}{ }^{7}(1-K \cos \phi)^{7 / 2}}
\end{aligned}
$$

Real frequency of waves can be obtained given by Tyagi et.al., ${ }^{34)}$

With $K=\frac{2 R r}{L_{0}{ }^{2}}$ and $L_{0}^{2}=R^{2}+r^{2}+h^{2}$ 
Niobium $(\mathrm{Nb})$ offers lower surface resistance $\mathrm{R}_{\mathrm{s}}$ at high lower critical field $B_{c}$ in the presence of large energy gap $\Delta$, however coherence length $\xi$ become shorter for higher critical field $\mathrm{B}_{\mathrm{c}}$ as shown by eq. $7 \& 8$

$$
\text { Rs }=\frac{A \omega^{2}}{T} \exp \left(-\frac{\Delta}{T}\right)
$$

Neglecting residual stresses

$$
\mathrm{Bc}=\frac{2 \Phi}{\pi d^{2}} \ln \frac{d}{1.07 \xi} \quad \mathrm{D}<\lambda
$$

Calculating transition temperature $\mathrm{T}^{\prime}{ }_{\mathrm{c}}$ affected by different parameters to a considerable value saves a lot of time and effort wasted by trial and error method. These estimated values provide a reliable control so as to accommodate experimental results. Particularly work of Martinis et al. ${ }^{37)}$ provide a simplified result of Usadel Equation ${ }^{38)}$ which in turn is based on Bardeen-CooperSchrieffer (BCS) theory of superconductivity, valid in the diffusive limit. Usadel equation for a superconductive state represented by function $\theta(\mathrm{x}, \mathrm{E})$ can be idealized as

$$
i E \sin \theta+\Delta(x) \cos \theta=0
$$

here this is approximated for uniform superconductor $\mathrm{x}$ in one dimension only. $\theta$ in the range of 0 to $\pi / 2$.

Where,

$\cos \theta$ is replaced by $\cos \theta_{B C S}=|E| / \sqrt{E^{2}-\Delta^{2}}$

and $\sin \theta$ by $\sin \theta_{B C S}=\Delta / \sqrt{\Delta^{2}-E^{2}}$

In superconducting region $\Delta$ is taken as constant. Taking clue from BCS gap equation

$$
T_{c}=T_{c o}\left[\frac{\alpha}{(1.13 \alpha+1.13) t}\right]^{\alpha}
$$

Where $\propto=d_{n} n_{n} / d_{s} n_{s}$, dn and ds are thickness of normal and superconducting film, $\mathrm{n}_{\mathrm{s}}$ and $\mathrm{n}_{\mathrm{n}}$ are density of electronic state in normal and superconducting film.

\section{Results and discussion}

To understand and demonstrate relative dependence of film thickness on Nitrogen concentration and to facilitate comparison of superconducting transition temperature and its range exhibiting superconductivity is computed and analysed with the help of mathematical model and computer technique by means of selectively chosen plasma parameters as shown in Table 1. Results are found in good proximity with literature.

Table 1. Coating procedure parameters - current 1-1.20 A, with deposition time 6- 60 per minute.

\begin{tabular}{|l|l|l|l|}
\hline T' ${ }_{C}(\mathrm{~K})$ & $\mathrm{N}$ (at. \%) & $\mathrm{N}_{2} \mathrm{P} \%$ & $\mathrm{Nb}$ \\
\hline 16.55 & 42.5 & 18 & 41.7 \\
\hline 16.67 & 46.1 & 21 & 37.7 \\
\hline
\end{tabular}

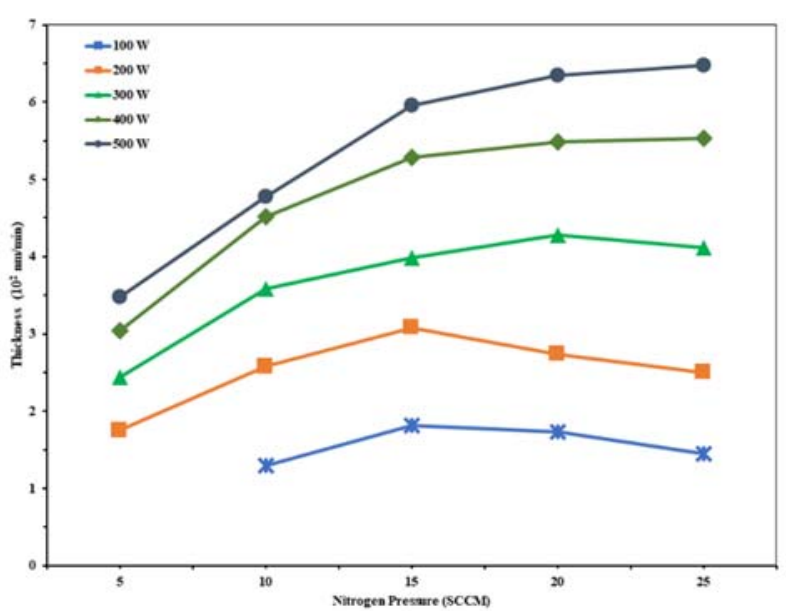

Fig. 2: Thickness versus SCCM nitrogen pressure

Fig. 2 shows thickness of $\mathrm{NbN}$ film at varied power densities under the influence of nitrogen pressure. Film thickness follows a steady increase and decrease after reaching an optimal thickness. After a critical range it presents insensitivity towards nitrogen in the deposition chamber.

As inferred by literature though plasma condition directly affect deposition of target material on a substrate and is also evident from the figure where at higher power substantially higher deposition rates are obtained. Maximum growth is achieved in a range of 13 to 19 SCCM, which observed approximately $18 \%$ growth in small window and almost getting stagnant and insensitive to further increase in nitrogen. ${ }^{27-29,39)}$ with increased power at a single point of observation that is at some fixed $\mathrm{N}_{2}$ partial pressures, thickness or growth rate enhances due to increase of sputtering rate $(\mathrm{Nb})$, further effecting on stability of $\mathrm{NbN}$ Phases due to increased $\mathrm{Nb} / \mathrm{N}$ ratio. This optimum window provide maximum variation in thickness of film as due to epitaxial strain superconducting properties get affected for very thin film.

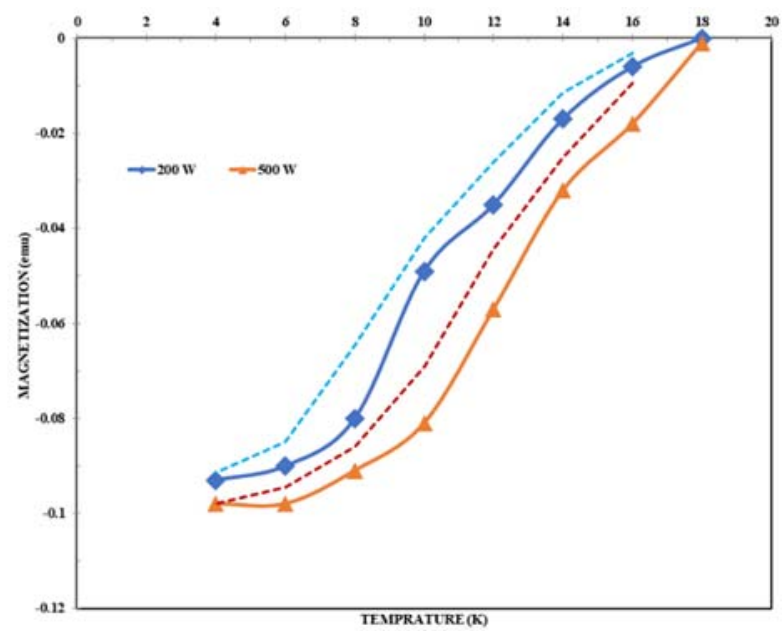

Fig. 3: Temp (K) versus magnetization showing superconductivity 
The influence of temperature on superconductivity is given in Fig. 3 Dashed lines are guiding line representing average mean of corresponding values. The computed results shows that their exists superconductivity at temperature ranging from $3 \mathrm{~K}$ to $14 \mathrm{~K}$. Superconductivity is analyzed and shown by the values as measure of magnetic moment at low temperature at subsequent range. Practically it is done by placing samples in constant magnetic field and then sample is demagnetized, and values obtained of magnetic moment and are analyzed. Our results confirm a close relationship between the shape of magnetic hysteresis loop obtained by Jirsa et. al and Mikitik et. al ${ }^{40,41)}$. The deviation from experimental Magnetic Hytresis loop (MHL) might be due to presence of phases and impurities at high fields. Increase in critical currents and activating non superconducting particles due to additional pinning and due to interplay of different pinning regimes might lead to fishtail phenomena ${ }^{42-45)}$. Free electron density could be a source of error in computed results. Different power intensity has not such a significance effect on superconducting properties, but a critical thickness exists that is must for presenting compositional superconductors. Figure 3 shows response close to diamagnetic response at temperature $\sim 8 \mathrm{~K}$ to $\sim 11.5 \mathrm{~K}$ as experimentally proved by Zou et al. ${ }^{46)}$ though not confirming exactly to their findings our reason for that is due to presence of $\delta-\mathrm{NbN}$ in hexagonal and cubic from where cubic $\delta-\mathrm{NbN}$ is mechanically unstable and criteria is not included in calculations.

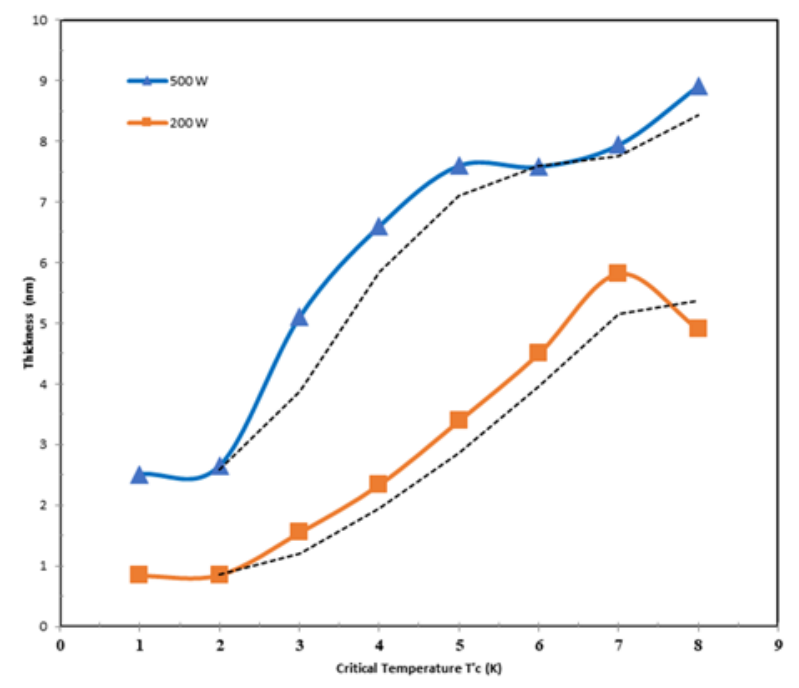

Fig. 4: Critical temperature ( $\left.T^{\prime} \mathrm{c}\right)$ versus thickness (nm)

Fig. 4 shows variation of critical temperature T'c at constant power but varying thickness of film, Dashed lines are guiding line representing average mean of corresponding values. considering conditions near stoichiometry from $2.3 \mathrm{~K}$ to $6.5 \mathrm{~K}$. as film growth showing insensitivity after certain pressures while taking case of maximum and minimum growth at a power density. The maximum T'c obtained is less than maximum critical temperature ${ }^{47)}$ at a perfect stoichiometric ratio though confirming the pattern and critical field at $\sim 4.4 \mathrm{~K}$. At fermi level T'c shows sensitivity due to phases and density of states and might be the reason for the change. The dependence of thickness over T'c for films were analysed by considering the electrical resistance as function of temperature, coherence length value for film is taken from electron mean-free path. For a narrow window range of transition temperature reversal of values is observed as shown confirming in good agreement to Destraz et.al., ${ }^{48)}$

It seems that instead of disordered scattering $T$ 'c, in different growth conditions and film, is governed by change in stoichiometric compound. While decrease in sputtering power there is an indication of decrease in T'c as with reducing power $\mathrm{Nb} / \mathrm{N}$ ratio is affected and carrier density as function of stoichiometric compound decreases.

Superconducting T'c goes down as the local density is suppressed by grain boundary ${ }^{49,50)}$ at different levels of T'c , by analyzing carrier density based on hall effect in thin film can be explored to resolve above issue not done here will be stated elsewhere. Transition temperature shows a similar behavior for a range of film thickness.

\section{Conclusion}

Here in this study thin films of $\mathrm{Nb}$ were deposited upon substrates at different $\mathrm{N}_{2}$ partial pressures and $\mathrm{NbN}$ films are investigated by computational technique using Usadel equation for a superconductive state. Film developed at 200W and 500W covers maximum range of nitrogen pressure and presents region for maximum film growth variation. Film at these power exhibits superconductivity in the range of $6.2 \mathrm{~K}$ to $13 \mathrm{~K}$ for the range of $0.078 \mathrm{emu}$ to $0.02 \mathrm{emu}$, while having most stability at higher power $500 \mathrm{~W}$. At higher fields due the presence of impurities and pinning effect an deviation from computed Magnetic Hytresis Loop is observed during experimental findings as probably by activation of non-superconducting particles and due to the influence of free electron density. Superconducting properties are not significantly affected by power density. $\mathrm{Nb}$ thickness reduced with decrease in superconductivity transition temperature. Film growth after certain pressures does not shows much dependence on the maximum critical temperature T'c whereas T'c maximum representing maximum growth is well below critical temperature at perfect stoichiometric ratio. This phenomenon is due to combined effect of film resistance, film structure and growth at particular thickness.

Thin $\mathrm{NbN}$ film grown over a substrate is probed by Usadel equation for close fitting to experimental data available. Usadel equation incorporating BCS theory of superconductivity relates decrease in film thickness to increase in BCS coherence length $\xi \sim 3.2 \mathrm{~nm}$ at a temperature approaching to critical value $14.5 \mathrm{~K}$ and magnetization value of 0.05 emu. Affecting stability of $\mathrm{NbN}$ Phases with increase of sputtering rate $(\mathrm{Nb})$ corresponding to respective $\mathrm{Nb} / \mathrm{N}$ ratio. 


\section{Nomenclature}

$\mathrm{Tp} \quad$ - $\quad$ Relative thickness of the film for planar magnetron sputtering

$\mathrm{T}_{\mathrm{c}} \quad$ - $\quad$ Relative thickness of the film for cylindrical magnetron sputtering

$\begin{array}{lll}\alpha & - & \text { Thermal velocity of ions } \\ R & - & \text { Radius of plasma column } \\ r & - & \text { Radius of plasma nozzle } \\ \mathrm{H} & - & \text { Substrate to target height } \\ \Phi & - & \text { Azimuthal angle } \\ \mathrm{Rs} & - & \text { Surface resistance } \\ \mathrm{Bc} & - & \text { Critical field } \\ \xi & - & \text { Coherence length } \\ \omega=\omega_{r}+i \gamma & -\end{array}$

$\mathrm{T}_{\mathrm{c}}{ }_{\mathrm{c}} \quad$ - $\quad$ Superconducting Transition

temperature

$E(x) \quad$ - $\quad$ Magnitude of homogeneous Direct Current electric field w.r.t distance $\mathrm{x}$ from origin

$E_{0 x} \quad$ - Magnitude of homogeneous Direct Current electric field at origin

$\begin{array}{lll}e_{s} & - & \text { Charge of species } \\ n, p & - & \text { Order of Bessel function } \\ x & - & \text { Distance from origin } \\ \Omega_{i} & - & \text { Gyro frequency } \\ Z(\xi) & - & \text { Plasma dispersion function }\end{array}$

\section{References}

1) J.I. Kodama, M. Itoh, and H. Hirai, "Superconducting transition temperature versus thickness of nb film on various substrates," J. Appl. Phys., 54 (7) 4050-4054 (1983). doi:10.1063/1.332534.

2) L. Xiao, X. Lu, D. Xie, W. Tan, Z. Yang, J. Zhao, D. Yang, and Y. Yang, "THE study of deposition method of nb3sn film on cu substrate," 18th Int. Conf. RF Supercond., 131-133 (2017). doi:10.18429/JACoWSRF2017-MOPB036.

3) A.E. Dane, A.N. McCaughan, D. Zhu, Q. Zhao, C.S. Kim, N. Calandri, A. Agarwal, F. Bellei, and K.K. Berggren, "Bias sputtered nbn and superconducting nanowire devices," Appl. Phys. Lett., 111 (12) (2017). doi:10.1063/1.4990066.

4) A. Gurevich, "Enhancement of rf breakdown field of superconductors by multilayer coating," Appl. Phys.
Lett., 88 (1) 1-4 (2006). doi:10.1063/1.2162264.

5) A. Matsumoto, H. Kitaguchi, T. Kuwahara, S. Sakai, T. Sueyoshi, and T. Fujiyoshi, "Microstructure and superconducting properties of doped bi,pb-2223 thin films fabricated by sputtering and annealing," IEEE Trans. Appl. Supercond., 28 (4) (2018). doi:10.1109/TASC.2018.2799424.

6) H. Naragino, M. Egiza, A. Tominaga, K. Murasawa, H. Gonda, M. Sakurai, and T. Yoshitake, "Fabrication of ultrananocrystalline diamond/nonhydrogenated amorphous carbon composite films for hard coating by coaxial arc plasma deposition," Evergreen, 3 (1) 1-5 (2016). doi:10.5109/1657379.

7) A. Maurya, and P.K. Jha, "Numerical investigation of in-mold electromagnetic stirring process for fluid flow and solidification," COMPEL - Int. J. Comput. Math. Electr. Electron. Eng., 36 (4) 1106-1119 (2017). doi:10.1108/COMPEL-10-2016-0460.

8) T. Polakovic, S. Lendinez, J.E. Pearson, A. Hoffmann, V. Yefremenko, C.L. Chang, W. Armstrong, K. Hafidi, G. Karapetrov, and V. Novosad, "Room temperature deposition of superconducting niobium nitride films by ion beam assisted sputtering," APL Mater., 6 (7) (2018). doi:10.1063/1.5031904.

9) A.E. Dane, and S.B.E. Sciences, "Reactive dc magnetron sputtering of ultrathin superconducting niobium nitride films," Master Sci.Thesis@ Harvard, (2010). http://hdl.handle.net/1721.1/97257.

10) P. Dhakal, S. Chetri, S. Balachandran, P.J. Lee, and G. Ciovati, "Effect of low temperature baking in nitrogen on the performance of a niobium superconducting radio frequency cavity," Phys. Rev. Accel. Beams, $21 \quad$ (3) $32001 \quad$ (2018). doi:10.1103/PhysRevAccelBeams.21.032001.

11) S. Wilde, B. Chesca, A.N. Hannah, D.O. Malyshev, O.B. Malyshev, S.M. Pattalwar, R. Valizadeh, and G.B.G. Stenning, "Physical vapour deposition of thin films for use in superconducting rf cavities," 3294 3252 (2015).

12) D.W. Kim, H.S. Kil, K. Nakabayashi, S.H. Yoon, and J. Miyawaki, "Improvement of electric conductivity of non-graphitizable carbon material via breakingdown and merging of the microdomains," Evergreen, 4 (1) 16-20 (2017). doi:10.5109/1808307.

13) H. Elserafy, "Assessment of demo reactors for fusion power utilization," Evergreen, 5 (4) 18-25 (2018). doi:10.5109/2174854.

14) Z. Han, X. Hu, J. Tian, G. Li, and G. Mingyuan, "Magnetron sputtered nbn thin films and mechanical properties," Surf. Coatings Technol., 179 (2-3) 188192 (2004). doi:10.1016/S0257-8972(03)00848-X.

15) X. Yang, L. You, L. Zhang, C. Lv, H. Li, X. Liu, H. Zhou, and Z. Wang, "Comparison of superconducting nanowire single-photon detectors made of nbtin and nbn thin films," IEEE Trans. Appl. Supercond., 28 (1) (2018). doi:10.1109/TASC.2017.2776288.

16) M. Kabiruzzaman, R. Ahmed, T. Nakagawa, and S. 
Mizuno, "Investigation ofc $(2 \times 2)$ phase of $\mathrm{pb}$ and bi coadsorption on $\mathrm{cu}(001)$ by low energy electron diffraction," Evergreen, 4 (1) 10-15 (2017). doi: $10.5109 / 1808306$.

17) G. Gupta, and R.K. Tyagi, "Investigation of Titanium as Thin Film Deposited Material Thereon Effect on Mechanical Properties," in: Adv. Ind. Prod. Eng., Springer, Singapore, 2019: pp. 315-323. doi:10.1007/978-981-13-6412-9_30.

18) A. Matsumoto, H. Kitaguchi, T. Kuwahara, S. Sakai, T. Sueyoshi, and T. Fujiyoshi, "Microstructure and superconducting properties of doped bi,pb-2223 thin films fabricated by sputtering and annealing," IEEE Trans. Appl. Supercond., 28 (4) 2-6 (2018). doi:10.1109/TASC.2018.2799424.

19) D.S. Katzer, N. Nepal, M.T. Hardy, B.P. Downey, D.F. Storm, E.N. Jin, R. Yan, G. Khalsa, J. Wright, A.C. Lang, T.A. Growden, V. Gokhale, V.D. Wheeler, A.R. Kramer, J.E. Yater, H.G. Xing, D. Jena, and D.J. Meyer, "Molecular beam epitaxy of transition metal nitrides for superconducting device applications," Phys. Status Solidi, 217 (3) 1900675 (2020). doi:10.1002/pssa.201900675.

20) N. Sayeed, U. Pudasaini, C.E. Reece, G. Eremeev, and H.E. Elsayed-Ali, "Structural and superconducting properties of nb 3 sn films grown by multilayer sequential magnetron sputtering," (2019). doi:10.1016/j.jallcom.2019.06.017.

21) A.C. De Oliveira, J. Lidovino, R.M. Oliveira, D. Aparecida, and P. Reis, "The influence of hightemperature nitrogen plasma-based ion implantation on niobium creep behavior," 2020 (2020).

22) S.G. Kim, N. Kim, H.S. Shim, O.M. Kwon, and D. Kwon, "Evaluation and control of mechanical degradation of austenitic stainless 310 s steel substrate during coated superconductor processing," Met. Mater. Int., $24 \quad$ (3) 448-454 (2018). doi:10.1007/s12540-018-0076-9.

23) M. Egiza, H. Naragino, A. Tominaga, K. Murasawa, H. Gonda, M. Sakurai, and T. Yoshitake, "Si and cr doping effects on growth and mechanical properties of ultrananocrystalline diamond/amorphous carbon composite films deposited on cemented carbide substrates by coaxial arc plasma deposition," Evergreen, 3 (1) 32-36 (2016). doi:10.5109/1657738.

24) S.K. Kim, and R. Hattori, "Study of contact resistance on organic thin-film transistor with surface treatments," Evergreen, 2 (1) 1-5 (2015). doi:10.5109/1500421.

25) G. Gupta, and R.K. Tyagi, “An experimental evaluation of mechanical properties and microstructure change on thin-film-coated aisi-1020 steel," Mater. Perform. Charact., 8 (3) 20180143 (2019). doi:10.1520/MPC20180143.

26) G. Gupta, R.K. Tyagi, S.K. Rajput, P. Saxena, A. Vashisth, and S. Mehndiratta, "PVD based thin film deposition methods and characterization / property of different compositional coatings - a critical analysis," Mater. Today Proc., (2020). doi:10.1016/j.matpr.2020.07.132.

27) A. Shoji, S. Kiryu, and S. Kohjiro, "Superconducting properties and normal-state resistivity of singlecrystal nbn films prepared by a reactive rf-magnetron sputtering method," Appl. Phys. Lett., 60 (13) 1624 1626 (1992). doi:10.1063/1.107220.

28) A. Kawakami, Z. Wang, and S. Miki, "Fabrication and characterization of epitaxial nbn/mgo/nbn josephson tunnel junctions," J. Appl. Phys., 90 (9) 4796-4799 (2001). doi:10.1063/1.1409583.

29) E.J. Cukauskas, "The effects of methane in the deposition of superconducting niobium nitride thin films at ambient substrate temperature," J. Appl. Phys., $54 \quad$ (2) 1013-1017 (1983). doi:10.1063/1.332117.

30) "Thin film processes ii - 1st edition," (n.d.). https://www.elsevier.com/books/thin-film-processesii/kern/978-0-08-052421-4 (accessed August 19, 2020).

31) M. Engel, Andreas and Semenov, Alexey and Hübers, Heinz-Wilhelm and Il'in, Konstantin and Siegel, "Electric noise and local photon-induced nonequilibrium states in a current-carrying nanostructured superconductor," New Front. Supercond. Res. Nov. Sci. Publ., 153-189 (2006). https://elib.dlr.de/45078/ (accessed August 19, 2020).

32) A. Semenov, B. Günther, U. Böttger, H.W. Hübers, H. Bartolf, A. Engel, A. Schilling, K. Ilin, M. Siegel, R. Schneider, D. Gerthsen, and N.A. Gippius, "Optical and transport properties of ultrathin nbn films and nanostructures," Phys. Rev. B - Condens. Matter Mater. Phys., $80 \quad$ (5) $054510 \quad$ (2009). doi:10.1103/PhysRevB.80.054510.

33) R.K. Tyagi, R.S. Pandey, and A. Kumar, "Surface coating by means of velocity shear instability in plasma," Theor. Found. Chem. Eng., 46 (5) 508-514 (2012). doi:10.1134/S0040579512050193.

34) R.K. Tyagi, K.K. Srivastava, and R.S. Pandey, "Analysis of electrostatic ion-cyclotron instability driven by parallel flow velocity shear," Surf. Eng. Appl. Electrochem., $47 \quad$ (4) $370 \quad$ (2011). doi:10.3103/S1068375511040144.

35) K.I. Grais, A.A. Shaltout, S.S. Ali, R.M. Boutros, K.M. El-behery, and Z.A. El-Sayed, "A new formula for sputtering yield as function of ion energies at normal incidence," Phys. B Condens. Matter, 405 (7) 1775-1781

(2010). doi:https://doi.org/10.1016/j.physb.2010.01.038.

36) X.Q. Meng, X.J. Fan, and H.X. Guo, “A new formula on the thickness of films deposited by planar and cylindrical magnetron sputtering," Thin Solid Films, 335 (1-2) 279-283 (1998). doi:10.1016/S00406090(98)00890-6.

37) J.M. Martinis, G.C. Hilton, K.D. Irwin, and D.A. Wollman, "Calculation of tc in a normal- 
superconductor bilayer using the microscopic-based usadel theory," Nucl. Instruments Methods Phys. Res. Sect. A Accel. Spectrometers, Detect. Assoc. Equip., $444 \quad$ (1) 23-27 (2000). doi:10.1016/S01689002(99)01320-0.

38) K.D. Usadel, "Generalized diffusion equation for superconducting alloys," Phys. Rev. Lett., 25 (8) 507509 (1970). doi:10.1103/PhysRevLett.25.507.

39) S.A. Wolf, I.L. Singer, E.J. Cukauskas, T.L. Francavilla, and E.F. Skelton, "Effects of deposition parameters on the properties of superconducting rf reactively sputtered nbn films," J. Vac. Sci. Technol., 17 (1) 411-414 (2002). doi:10.1116/1.570469.

40) D.D. and M.R.K. M. Jirsa, L. Pu ${ }^{\circ}$ st, "Fishtail shape in the magnetic hysteresis loop for superconductors: interplay between different pinning mechanisms," Phys. Rev. B, 55 (5) 3276-3284 (1997). https://www.researchgate.net/publication/215596325 _Fishtail_shape_in_the_magnetic_hysteresis_loop_f or_superconductors_Interplay_between_different_pi nning_mechanisms.

41) G.P. Mikitik, and E.H. Brandt, "Peak effect, vortexlattice melting line, and order-disorder transition in conventional and high-(formula presented) superconductors," Phys. Rev. B - Condens. Matter Mater. Phys., $64 \quad$ (18) $184514 \quad$ (2001). doi:10.1103/PhysRevB.64.184514.

42) V.I. Ivashchenko, P.L. Skrynskii, O.S. Litvin, A.D. Pogrebnjak, V.N. Rogoz, G. Abadias, O. V. Sobol', and A.P. Kuz'menko, "Structure and properties of nanostructured nbn and nb-si-n films depending on the conditions of deposition: experiment and theory," Phys. Met. Metallogr., 116 (10) 1015-1028 (2015). doi:10.1134/S0031918X15080062.

43) V.N. Zhitomirsky, I. Grimberg, L. Rapoport, N.A. Travitzky, R.L. Boxman, S. Goldsmith, A. Raihel, I. Lapsker, and B.Z. Weiss, "Structure and mechanical properties of vacuum arc-deposited nbn coatings," Thin Solid Films, 326 (1-2) 134-142 (1998). doi:10.1016/S0040-6090(98)00544-6.

44) N. Cansever, M. Danişman, and K. Kazmanli, "The effect of nitrogen pressure on cathodic arc deposited nbn thin films," Surf. Coatings Technol., 202 (24) 5919-5923 doi:10.1016/j.surfcoat.2008.06.158.

(2008).

45) W. Lengauer, M. Bohn, B. Wollein, and K. Lisak, "Phase reactions in the nb-n system below $1400^{\circ} \mathrm{c}$," Acta Mater, $48 \quad$ (10) 2633-2638 (2000). doi:10.1016/S1359-6454(00)00056-2.

46) Y. Zou, X. Qi, C. Zhang, S. Ma, W. Zhang, Y. Li, T. Chen, X. Wang, Z. Chen, D. Welch, P. Zhu, B. Liu, Q. Li, T. Cui, and B. Li, "Discovery of superconductivity in hard hexagonal €-nbn," Sci. Rep., 6 (February) 19 (2016). doi:10.1038/srep22330.

47) M.W. Williams, K.M. Ralls, and M.R. Pickus, "Superconductivity of cubic niobium carbo-nitrides," J. Phys. Chem. Solids, 28 (2) 333-341 (1967). doi:10.1016/0022-3697(67)90129-1.

48) D. Destraz, K. Ilin, M. Siegel, A. Schilling, and J. Chang, "Superconducting fluctuations in a thin nbn film probed by the hall effect," Phys. Rev. B, 95 (22) 1-6 (2017). doi:10.1103/PhysRevB.95.224501.

49) J.H. Tyan, and J.T. Lue, "Grain boundary scattering in the normal state resistivity of superconducting nbn thin films," J. Appl. Phys., 75 (1) 325-331 (1994). doi:10.1063/1.355853.

50) A. Nigro, G. Nobile, M.G. Rubino, and R. Vaglio, "Electrical resistivity of polycrystalline niobium nitride films," Phys. Rev. B, 37 (8) 3970-3972 (1988). doi:10.1103/PhysRevB.37.3970. 